\title{
LA-UR- 09-00019
}

Approved for public release;

distribution is unlimited.

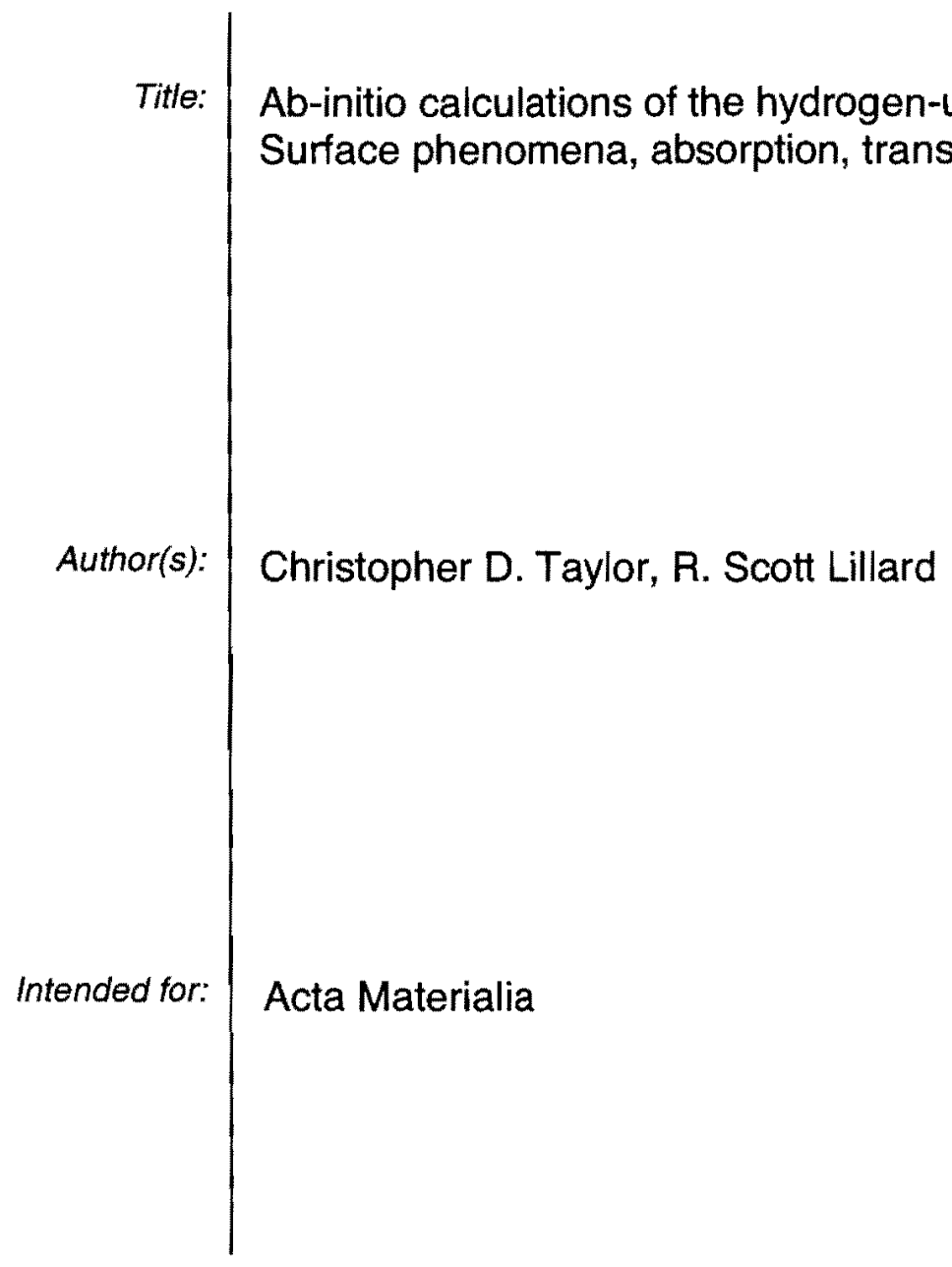

\section{. \\ - Los Alamos NATIONAL LABORATORY}

EST.1943

Los Alamos National Laboratory, an affirmative action/equal opportunity employer, is operated by the Los Alamos National Security, LLC for the National Nuclear Security Administration of the U.S. Department of Energy under contract DE-AC52-06NA25396. By acceptance of this article, the publisher recognizes that the U.S. Government retains a nonexclusive, royalty-free license to publish or reproduce the published form of this contribution, or to allow others to do so, for U.S. Government purposes. Los Alamos National Laboratory requests that the publisher identify this article as work performed under the auspices of the U.S. Department of Energy. Los Alamos National Laboratory strongly supports academic freedom and a researcher's right to publish; as an institution, however, the Laboratory does not endorse the viewpoint of a publication or guarantee its technical correctness. 
Ab-initio Calculations of the Hydrogen-Uranium System. Part I: Surface Phenomena, Absorption, Transport and Trapping

Christopher D. Taylor and R. Scott Lillard

Los Alamos National Laboratory

Los Alamos, NM 87544

Phone: (505) 667-5499; (505) 667-6325

Fax: (505) 667-2264

Email: cdtavlor@lanl.gov; lillard@lanl.gov

Last Modified:

November $24^{\text {th }}, 2008$ 


\begin{abstract}
Density functional theory calculations have recently been validated for $\alpha$-uranium $(\alpha-U)$ metal
\end{abstract} and, in this work, applied to the initial steps of uranium hydriding: surface phenomena, absorption into the bulk, bulk transport (diffusion) and trapping at defect sites. We investigate the surface chemistry of hydrogen $(\mathrm{H})$ on the (001) surface of $\alpha-\mathrm{U}$, and the influence of high-coordinate geometries on $\mathrm{H}_{2}$ adsorption and dissociation on the metal surface. In the adsorbed state $\mathrm{H}$ already has a partially ionic character. Although $\mathrm{H}$ adsorbs exothermically to the (001) surface, with respect to the reference state of $\mathrm{H}_{2}$, we find that it is endothermic to absorb $\mathrm{H}$ into the bulk, with off-center octahedral absorption (i.e. a square-pyramidal coordination of $\mathrm{H}$ in the lattice) having the lowest absorption energy of $0.39 \mathrm{eV}$ relative to molecular $\mathrm{H}_{2}$. $\mathrm{H}$ absorption in interstitial sites is calculated to cause a local softening of the bulk modulus. The diffusion barrier for $\mathrm{H}$ in unstrained $\alpha-\mathrm{U}$ is calculated to be $0.6 \mathrm{eV}$. The energy of $\mathrm{H}$ absorption in a site adjacent to the chemical impurities $\mathrm{C}, \mathrm{S}, \mathrm{Si}$ was lowered by an amount proportional to the atomic radii of the impurity atom, and the resulting lattice strain $\mathrm{Si}>\mathrm{S}>\mathrm{C}$. Thus, impurities may promote hydriding by providing surfaces or pre-strained zones for $\mathrm{H}$ uptake. 


\section{Introduction}

The $\alpha$-uranium ( $\alpha-\mathrm{U}$, orthorhombic) to uranium hydride $\left(\mathrm{UH}_{3}\right.$, cubic) phase transformation (often referred to as $\mathrm{U}$ corrosion) involves both a change in chemical composition and structure. In this process it is generally accepted that six unique phenomena can be identified: 1) surface phenomena, 2) absorption, 3) transport, 4) trapping, 5) saturation and, finally, 6) phase transformation (Figure 1). ${ }^{1}$ In this paper we will address the first four processes: surface phenomena, absorption into the $\alpha$-U lattice, transport in the bulk and trapping by defects. In a subsequent paper, we consider saturation and phase transformation.

Step 1, broadly described as surface phenomena, consists of (a) mass transport of molecular $\mathrm{H}_{2}$ to the metal surface, (b) the dissociation of molecular $\mathrm{H}_{2}$ to form chemisorbed atomic hydrogen $(\mathrm{H})$, and (c) surface migration and (d) absorption into the bulk. While surface analytical studies of hydrogen interactions with many metal surfaces have been quite successful, $H$ interactions with clean $\alpha$-U surfaces have been constrained by relatively fast oxide and hydride reaction rates. Thus, only limited information about U-H surface interactions from an atomistic or kinetics standpoint is known. In thermal desorption spectroscopy experiments, Balooch et al. report that the sticking probability (coverage/exposure) for $\mathrm{H}_{2}$ on polycrystalline $\alpha-\mathrm{U}$ is 0.04 at room temperature and a coverage of $9 \times 10^{17}$ molecules $/$ mole. $^{2}$ Assuming complete dissociation this results in a saturation coverage of approximately $0.15 \mathrm{H}$ atoms / $\mathrm{U}$ atom. Based upon results from direct recoil spectroscopy 
experiments, Swissa reports a sticking probability of 0.1 although the model assumptions and concentration of adsorption sites used to derive this value limit its usefulness. ${ }^{3}$

In step 2, absorption of $\mathrm{H}$ into the bulk is followed by diffusion to trap sites where saturation occurs. Mallet determined the diffusion coefficient (D) of $\mathrm{H}$ in $\alpha-\mathrm{U}$ using the degassing rate and a solution of law for cylinders. ${ }^{4}$ It was shown that the diffusivity of $\mathrm{H}$ in $\alpha$-U followed the relationship $\log (D)=-2420 / T-1.71$ where $\mathrm{T}$ is temperature in Kelvins and $\mathrm{D}$ is in $\mathrm{cm}^{2} / \mathrm{s}$. This Arrhenius relationship for the diffusion constant leads to an estimate of $0.49 \mathrm{eV}$ for the diffusion barrier of $\mathrm{H}$ in $\alpha$ U. Accordingly, the diffusivity of $\mathrm{H}$ in the bulk is quite low in comparison to other metals at this temperature (at $\mathrm{T}=573 \mathrm{~K}\left(300^{\circ} \mathrm{C}\right) \mathrm{D}$ is $\left.1.16 \times 10^{-6} \mathrm{~cm}^{2} / \mathrm{s}\right)$. At room temperature the value is exceedingly small, $1.5 \times 10^{-10} \mathrm{~cm}^{2} / \mathrm{s}$.

The solubility of $H$ in $\alpha-U$ is also low, indicating that lattice sites for hydrogen possess a high chemical potential, and that trap sites must be in small concentrations. Studies by Mallet at 1 atm $\mathrm{H}_{2}$ and $300^{\circ} \mathrm{C}$ found the solubility of $\mathrm{H}$ in $\alpha-\mathrm{U}$ to be $0.2 \mathrm{ppm}\left(9.3 \times 10^{-5}\right.$ atoms $\mathrm{H} / \mathrm{unit}$ cell $\left.\mathrm{U}\right){ }^{4}$ Mattraw reported that the low-pressure solubility obeyed the relationship $S=K \sqrt{P}$ where $\mathrm{S}$ is solubility in cc/g $\mathrm{U}$, $\mathrm{P}$ is pressure in $\mathrm{mm} \mathrm{Hg}$, and $\mathrm{K}$ is the solubility constant the value of which is $3.3 \times 10^{-3} .^{5}$ Thus, for $295^{\circ} \mathrm{C}$ we calculate from the Mattraw relationship a solubility at $\mathrm{P}=100 \mathrm{mmHg}\left(1.3 \times 10^{-4} \mathrm{~atm}\right)$ of $\mathrm{S}=$ $0.033 \mathrm{cc} \mathrm{H}_{2} / \mathrm{g} \mathrm{U}$, or $3 \mathrm{ppm}\left(1.4 \times 10^{-3}\right.$ atoms $\mathrm{H} /$ unit cell $\left.\mathrm{U}\right)$; higher than the Mallet data but still far below the U:H ratio of $1: 3$ in the hydride (a concentration equivalent to 12.5 parts-per-thousand, ppt). 
The low diffusivity and solubility of $\mathrm{H}$ in $\alpha-\mathrm{U}$ may explain the predominance of near-surface hydriding and the inability to measure diffusivities using permeation techniques. The low solubility of $\mathrm{H}$ in $\mathrm{U}$ also points out the need and likely role for trap sites for the aggregation of $\mathrm{H}$ atoms.

In this work, we have applied density functional theory (DFT) to investigate the interactions between metallic $\alpha-U$ and molecular, as well as atomic, $\mathrm{H}$ at the atomic scale such that we may identify the critical atomistic steps associated with the uranium-hydriding process. This computational method has been applied recently to a number of metal-H systems, including $\mathrm{Al}^{6}{ }^{6} \mathrm{Fe}^{7} \mathrm{Zr}_{,}^{8} \mathrm{Ti}^{9}{ }^{9} \mathrm{Ta}$ and $\mathrm{Nb},{ }^{10}$ as well as surfaces. ${ }^{11}$ Electronic structure calculations of the ${\mathrm{U}-\mathrm{UH}_{3}}_{3}$ have been performed by Switendick, ${ }^{13}$ and Balasubramanian. ${ }^{14}$ The former study, while shedding light on the electronic configuration of $U$ in $\mathrm{UH}_{3}$, was constrained to extreme simplifications by computational limitations, and thus did not tackle the question of atomic rearrangement. ${ }^{13}$ The latter study took an atom-centric approach to the investigation of $\mathrm{UH}_{3}$ formation mechanisms (i.e. atomic $\mathrm{U}$ inserting into a $\mathrm{H}_{2}$ molecule, rather than a solid uranium surface) ${ }^{14}$ neglecting the important solid-state steps of adsorption, absorption and phasetransformation, which we seek to elucidate here in this work. Very recently a detailed study of molecular hydrogen interactions with $\alpha-\mathrm{U}(001)$ has been published, with results and methods that support the calculations reported in this work. ${ }^{15}$ Whereas this latter paper focuses closely on mechanisms for $\mathrm{H}_{2}$ dissociation over $\alpha-\mathrm{U}(001)$, herein we apply ourselves more closely to developing an overall mechanistic understanding of the hydriding process, as described in the steps above. 
The present computational model is based on the projector augmented wave (PAW) potential for

U developed by Kresse et al. ${ }^{16}$. PAW calculations for $\alpha$-U have been published elsewhere and are comparable in accuracy to the full-potential calculations reported by others. ${ }^{17,18}$ With this model we examine the dissociation of molecular $\mathrm{H}_{2}$ on an $\alpha-\mathrm{U}(001)$ surface to form chemisorbed $\mathrm{H}$, the adsorption to absorption transition, diffusion, trapping at vacancies, and the influence of the interstitial impurities carbon (C), silicon ( $\mathrm{Si}$ ), and sulfur ( $\mathrm{S}$ ) on $\mathrm{H}$ binding energies (absorption). 


\section{Computational Methods}

The electronic structure problems related to the first-principles computation of the interactions between $\mathrm{H}$ and $\alpha-\mathrm{U}$ surfaces, lattice sites and defect states described herein were solved using the efficient, parallelized electronic structure code Vienna Ab-initio Simulation Package (VASP). ${ }^{19}$ The projector augmented wave (PAW) functions for uranium, $\mathrm{H}$ and the candidate impurity atoms $(\mathrm{C}, \mathrm{Ne}, \mathrm{S}$, Si and O) supplied with version 4.6 of VASP were used without modification. The PW91 exchangecorrelation functional was used, except when noted for certain comparisons performed with the LDA functional. $^{20}$ Tests of the energy convergence with respect to the k-point mesh size and energy cut-off led to the choice of an energy cut-off of $500 \mathrm{eV}$ and a $\Gamma$-centered Monkhorst-Pack k-point mesh generated using a generating length of $40 \AA^{21}$ This choice of k-point mesh corresponds to a $14 \times 7 \times 8$ mesh for the conventional 4-atom orthorhombic unit cell of $\alpha$-U (Figure 2). The resulting energies were converged with respect to energy cut-off and k-point mesh to within $2 \mathrm{meV}(0.15 \mathrm{mRy})$. To perform relaxations the Methfessel-Paxton smearing method was used to provide more accurate forces (width of $0.2 \mathrm{eV}$ ), whereas for single-point calculations the Bloechl tetrahedron method was adopted. ${ }^{22,23}$ Selfconsistent electronic structure calculations were iterated to within $0.1 \mathrm{meV}$, and, for geometric relaxations, iterations over the lattice positions were performed until forces were less than $0.05 \mathrm{eV} / \AA$. The relaxations were performed using the conjugate gradient method. 
Surfaces were created using the optimized lattice parameters, with full relaxation of the internal parameters and the interlayer spacings normal to the surface. The periodic slab model was used to treat the $U$ surfaces. In this model a finite number of layers of metal atoms was used to represent the semiinfinite metal, and these 'slabs' were then separated by a region of vacuum thick enough to minimize the interslab interactions. Because only a finite number of layers was used, and because this number must typically be small for the simulation to be computationally efficient, it was important to vary the number of layers required to obtain converged surface properties. Previously, we considered the variation of $\alpha$ U surface properties in the (001) direction for models consisting of between 3 and 7 atomic layers. ${ }^{17}$ In that study a 16x16x1 k-point mesh was used for the primitive $1 \times 1$ surface periodic unit cell. It was found that choosing three layer slabs led to errors of $0.04 \mathrm{eV}$ in the surface energy relative to the converged value at 5 layers. As these errors are within the reasonable limits expected from DFT calculations, and substantial computational savings are achieved by a three layer slab model, we utilize herein a three layer slab model for the U-H surface system. Furthermore, this choice is consistent with other surface slab calculations performed for hydrided surfaces in the literature. ${ }^{15,24}$

The energy of absorbed hydrogen at lattice interstitials, impurity sites and vacancies was determined using a 96 atom unit cell, of dimensions $(4 a, 2 b, 3 c)$, where $(a, b, c)$ are the lattice parameters of the conventional 4-atom unit cell. For this calculation the $\Gamma$-point was used to represent the Brillouin 
zone, which has an estimated accuracy of $0.05 \mathrm{eV}$ per hydrogen atom, when compared to calculations of the hydrogen absorption energy made with finer k-point meshes.

Scalar-relativistic calculations were performed for all systems, in which case the PAW-core contribution is considered to supply the leading relativistic correction to the electronic structure. Some validation of spin-orbit and scalar-relativistic methods was made for $\alpha-\mathrm{U}$ as well as the $\mathrm{UH}_{3}$ systems (see also our previous publication) ${ }^{17}$. Errors ensuing from neglecting the more complete treatment of spin-orbit interaction were less than $0.10 \mathrm{eV}$, within the expected limit of accuracy provided from DFT and most quantum-chemical methods (best estimates for this value are around $0.15 \mathrm{eV}$ ). ${ }^{25}$ 


\section{Results and Discussion}

\section{The dissociation, adsorption and transport of $H$ on the (001) $\alpha-U$ surface}

To initiate a hydriding reaction, $\mathrm{H}$ is dissociated, or activated, from the host molecule $\left(\mathrm{H}_{2}\right.$ or $\mathrm{H}_{2} \mathrm{O}$, for example) at the surface, from which point it may either diffuse atomically into and through the material, or immediately react. ${ }^{\prime}$ Herein we consider the reactivity of the $\alpha-U(001)$ surface, which we determined to be the lowest energy, low-index cleavage plane, towards atomic and molecular $\mathrm{H}_{2}$, with the goal of elucidating the role of the surface in hydriding kinetics.

$\mathrm{H}_{2}$ molecules approaching the $\alpha-\mathrm{U}(001)$ surface will be attracted to the surface, at first via weak, van der Waals interactions, but then, as the electronic states begin to overlap, by a chemisorption effect. This latter chemisorption is well captured within our calculations, as is revealed by the energy versus distance curves plotted in Figure 3. These curves were generated for the chemisorption of $\mathrm{H}_{2}$ positioned over an atop site, with the $\mathrm{H}$ atoms oriented towards the 3 -fold sites on either side of the surface $\mathrm{U}$ atom. Although the angles are different, and the triangles formed on the surface are not regular (rather they are isosceles due to the symmetry of the lattice), there exists an ABAB stacking sequence, and a series of 3 fold hollow sites that allow us to use a similar nomenclature as adopted for close-packed metal systems in the (111) plane sequence. There are therefore two inequivalent 3-fold sites, which are herein labeled e-hcp and e-fcc, where 'e' indicates an approximate equivalence to the hcp hollow site on close-packed

surfaces. As can be seen by the minimum in the potential energy at $2.35 \AA$, this configuration has 
a chemisorption energy of $-0.11 \mathrm{eV} / \mathrm{H}_{2}$ molecule. As the $\mathrm{H}_{2}$ molecule thermally oscillates in this chemisorption well, at a rate dependent upon the thermal energy of the molecule, it may crossover to the dissociated state, also shown in Figure 3, in which the two $\mathrm{H}$ atoms are bonded at the e-fcc and e-hcp sites adjacent to the atop site. This dissociated state has a significantly lower energy, and the crossover point at $2.15 \AA$ possesses a low barrier relative to the physisorption state of $0.05 \mathrm{eV} / \mathrm{H} 2$, or $0.025 \mathrm{eV} / \mathrm{H}$ atom. These energies are on the order of $\mathrm{kT}$ at room temperature $(0.03 \mathrm{eV})$, and hence there is essentially no barrier for $\mathrm{H}_{2}$ dissociation on the $\alpha-\mathrm{U}(001)$ surface at room temperature. It is certainly lower than the $26 \mathrm{kcal} / \mathrm{mol}$ determined from quantum chemical calculations of $\mathrm{U}$ atom insertion into $\mathrm{H}_{2}{ }^{14}$ and the experimentally determined range of apparent hydriding activation energies $(0.24-0.96 \mathrm{eV} /$ H-atom). From these results we conclude that dissociation over the $\alpha$-U(001) surface is not rate determining. ${ }^{26}$

$\mathrm{H}$ atoms are not restricted to adsorption at these 3-fold hollow sites, although $\mathrm{H}$ atoms typically prefer high coordinate sites on many metallic surfaces (for example, $\mathrm{Pu})^{24}$. Consistent with this trend, the adsorption energies for $\mathrm{H}$ on the (001) surface of $\alpha-\mathrm{U}$ are found to increase with increasing metal-H coordination. The energies of adsorption at the various sites illustrated in Figure 4 are presented in Table 1. It can be seen that, relative to $\mathrm{H}_{2}, \mathrm{H}$ adsorption is endothermic at the atop site, but moves to lower energies, with exothermic adsorption on the multi-coordinate sites. The two-fold sites have an adsorption energy of about $-0.3 \mathrm{eV}$, and the three-fold sites an adsorption energy of about $-0.5 \mathrm{eV}$. 
These energies are consistent with adsorption energies for $\mathrm{H}$ on the strongly $\mathrm{H}$-adsorbing noble and transition metals, such as $\mathrm{Pd}, \mathrm{Pt}, \mathrm{Ni}, \mathrm{Co}$ and $\mathrm{Re} .{ }^{27}$ The atomic adsorption energy of $-2.83 \mathrm{eV}$ at the e-fcc site is slightly larger than the energy for adsorption at the 3 -fold hollow site on $\mathrm{Pu}$, which is $-2.76 \mathrm{eV}{ }^{24}$

The charge state of $\mathrm{H}$ at the surface can be estimated by performing a topological analysis of the charge using Bader's Atoms in Molecules (AIM) technique. ${ }^{28,29}$ It is found that there is already significant charge transfer from $\mathrm{U}$ to $\mathrm{H}$ in the adsorbed state, with a charge of -0.5 electrons passing to the $\mathrm{H}$ atom. As will be seen later in this paper, the $-1 / 2$ charge state of $\mathrm{H}$ occurs frequently in $\mathrm{U}-\mathrm{H}$ systems (for clarity, it should be noted that the AIM charge state of $\mathrm{H}$ in the $\mathrm{H}_{2}$ molecule take on the physically sensible value of 0 ).

While it is likely that $\mathrm{H}$ will interact with steps/kinks or other defects on the surface, there is no experimental or computational evidence that the adsorption energies associated with these sites would be larger than a defect free surface. Further, we anticipate that they will be lower. Therefore, we postpone investigation of $\mathrm{H}$ interaction with surface features like kinks and steps for future work, as computation of extended surfaces is computationally expensive and is expected to have little impact on our conclusions. Instead we focus on the barrier to hydrogen passage across the $\alpha-U(001)$ surface. It is possible to freeze the $\mathrm{H}$ atom at a point which is coplanar with the $\mathrm{U}(001)$ surface, i.e. to consider a state that is intermediate to the penetration of the $\alpha$ - $\mathrm{U}$ lattice by $\mathrm{H}$, and to subsequently relax the positions of the surrounding uranium atoms. The energies for $\mathrm{H}$ passing through such sites can then be 
estimated and these energies have been tabulated herein as entry-d and entry-e in Table 1, according to the particular 3-fold site transgressed; c.f. Figure $4(\mathrm{~d}, \mathrm{e})$. The energy cost to transgression at site $\mathrm{d}$ is lower than at site e, presumably due to the bonding with sub-surface $U$ that occurs during the $d$-site entry. The computed barrier is $0.45 \mathrm{eV}$ for the $\mathrm{d}$ entry and $0.80 \mathrm{eV}$ for the e entry. These barriers are higher than the experimental hydriding kinetic measurements of Bloch where activation barriers, $E_{a}$, of between $0.24-0.39 \mathrm{eV}$ were observed. ${ }^{26}$ Therefore it is likely that defects (grain-boundaries, surfacing dislocations, interfaces with second phase particles, etc.) provide the dominant pathway for $\mathrm{H}$ entry into the material.

\section{Interstitial lattice sites for $H$ in orthorhombic $\alpha-U$ and related transport properties}

In this section we present the results of our investigation of $\mathrm{H}$ states with the $\alpha-\mathrm{U}$ lattice. We begin by describing the general topology of the perfect crystal lattice, and then proceed to discuss the absorption energies and binding configurations of various high symmetry absorption sites. We also present data relevant to the determination of $\mathrm{H}$ diffusion constants in $\alpha-\mathrm{U}$, the influence of dissolved $\mathrm{H}$ on the mechanical and cleavage properties of $\alpha-\mathrm{U}$, and the charge states for $\mathrm{H}$ bound to the lattice. As mentioned previously, when viewed in the (001) orientation, the $\alpha$-U lattice resembles an hcp type structure, and thus possesses analogous octahedral and tetrahedral interstices within the lattice. The anisotropy of these sites, however, is considerable. The octahedral site is elongated uniaxially, 
with a height:width ratio of 1.34 (regular octahedra have a ratio of 1). The central 'square' of the octahedron constrains the interstitial atom to smaller U-X distances in the plane (1.972 $\AA$ ), whereas the out of plane distances along the stretched axis are very large $(2.642 \AA)$. Octahedral absorption (oct, Figure 5a), therefore, requires significant rearrangement of the $U$ atom positions to optimize bonding. An optimal U-H bond length may be estimated from the covalent radii to be $1.76 \AA ;^{30}$ direct DFT computations of the interstitials indicate a preference for bond lengths closer to $2.1 \AA$, which, in fact, agrees better with values obtained from tabulated atomic radii. ${ }^{31}$ Symmetry breaking to one of the two square-pyramidal (sqpy) sites located above and below the centroid of the octahedron is found to result in a lower energy configuration with the optimal U-X distances of $2.11 \AA$ (Figure 5b). The tetrahedral sites (tet), while also being significantly distorted from a regular tetrahedral environment, possess metalcentroid bond lengths of $1.9 \AA$ (Figure $5 \mathrm{c}$ ). In Figure $5 \mathrm{~d}$ we illustrate the transition state for diffusion joining the octahedral/sqpy and tetrahedral sites.

We find that $\mathrm{H}$ absorption at the octahedral site is most endothermic, followed by the tetrahedral site, and then the square pyramidal site. The energies are all endothermic with respect to molecular $\mathrm{H}_{2}$, $0.53 \mathrm{eV}$ for octahedral, $0.42 \mathrm{eV}$ for tetrahedral and $0.39 \mathrm{eV}$ for the square pyramidal. The barrier for diffusion from the sqpy to the tet site is calculated herein as $0.62 \mathrm{eV}$. See the tabulated values in Table 1. Although in the unstrained state the tet site allows smaller $\mathrm{U}-\mathrm{H}$ bond lengths, clearly the tet configuration allows a better accommodation of $\mathrm{H}$ than the oct site. 
Since these values for absorption into the lattice (a potential intermediate in the hydride

formation process) exceed the range of experimentally determined barriers for hydride formation (0.24$0.39 \mathrm{eV}$ ), it is likely that that absorption occurs via strained or defect regions. We therefore proceed to calculate the energy of $\mathrm{H}$ absorption at the square pyramidal site for strained lattices. The binding energy for $\mathrm{H}$ as a function of total hydrostatic lattice strain is presented in Figure 6 (positive strains indicate tension, negative strains are compression). The binding energy drops significantly as the lattice is strained in tension, implying that $\mathrm{H}$ uptake at defects like dislocations and grain-boundaries will be significantly higher. Even at hydrostatic strains $<+0.5 \%$ the absorption energy drops to the $0.26 \mathrm{eV}$ required by kinetic limitations. A similar enhancement of $\mathrm{H}$ absorption with tensile strain has been evaluated theoretically for the $\mathrm{Ni}(111)$ surface. ${ }^{11}$

$\mathrm{H}$ absorption in the lattice also softens the bulk modulus of the material, leading to increased elasticity. This information can be obtained from the curvature of Figure 6. Without $\mathrm{H}$ present the bulk modulus is calculated to be $134 \mathrm{GPa}$ at this level of theory; when hydrogen is present at a concentration of 1.04 at. $\%$ the bulk modulus is calculated to be $128 \mathrm{GPa}$.

Insertion of $\mathrm{H}$ between planes of the lattice reduces the cohesive strength between those planes.

Hence $\mathrm{H}$ absorption also reduces the energy required for plane cleavage (the surface energy of the $(001)$ plane is $\left.1.38 \mathrm{~J} / \mathrm{m}^{2}\right)^{17}$. This phenomenon, elucidated in more detail by Van der Ven, ${ }^{32}$ occurs because $\mathrm{H}$ binding to a surface plane of $\alpha-U$ is stronger than $H$ binding to the lattice; in the case of the (001) 
surface this amount is $0.92 \mathrm{eV}$. Assuming a coverage of $1 \mathrm{H}$ atom per $9 \AA^{2}$, a lowering of the cleavage energy by $1.7 \mathrm{~J} / \mathrm{m}^{2}$ is possible, making crack formation now an exothermic process.

With respect to charge, dissolved $\mathrm{H}$, as well as the surface $\mathrm{H}$, possesses some ionic character, indicating that the $\mathrm{U}-\mathrm{H}$ interactions involve charge transfer. Similarly to the surface adsorption state, $\mathrm{H}$ in the lattice possesses a charge of $-0.5|\mathrm{e}|$. The fact that this is contributed from at least 4 metal atoms, compared to three in the surface state, implies that the charge transfer in the surface state leads to a greater polarization than occurs within the lattice (i.e. each atom of $\mathrm{U}$ in the surface state bound to $\mathrm{H}$ contributes $\sim 1 / 6 \mathrm{e}$, whereas in the absorption states each atom contributes less charge ( $1 / 8 \mathrm{e}$ for tet, $1 / 10$ e for sqpy, and 1/12e for octahedral, assuming equal contributions per atom).

To summarize, hydrogen absorption is an endothermic process, leading to low solubilities for $\mathrm{H}$ in $\alpha-U$. Thus hydrogen absorption can lead to enhanced cracking, and a softer bulk modulus of the metal. Furthermore, our calculations show that hydrogen in the lattice will be drawn to regions of lower chemical potential, such as presented by regions of tensile strain. Since the energy to bind hydrogen at regular interstitial sites in $\alpha-U$ is much higher than the activation barrier to the hydriding reaction, we postulate that hydriding does not involve these sites, but must involve regions of local lattice strain.

\section{Trapping of $H$ at point-defects in the lattice: vacancies and chemical impurities}


In this section we study the interaction of $\mathrm{H}$ with the strain fields that may result from interstitial impurities, such as $\mathrm{C}, \mathrm{S}$, and $\mathrm{Si}$, which provides some insight concerning the role of these impurities in hydride formation. ${ }^{33}$ In addition we consider how vacancies may attract $\mathrm{H}$ and partake in the total consideration of $\mathrm{H}$ interactions with bulk $\alpha$-U. Similar studies of $\mathrm{H}$-vacancy interactions have shown them to be significant for the $\mathrm{H}$ embrittlement of $\mathrm{Fe},{ }^{34}$ and $\mathrm{Al},{ }^{35}$ as well as other metals. ${ }^{36}$

We have considered the uptake of various impurity atoms in $\alpha$-U, placing the impurities at octahedral sites, which leads to significant lattice rearrangement, whereby the impurity atom pushes out the $U$ atoms bounding the central square plane of the octahedron to optimize the metal-impurity bond lengths. We quantify this local distortion, $\sigma$, as the average distortion in U-U bond distance connecting the nearest neighbors to the impurity:

$$
\sigma=\sum \frac{r_{u U}-r_{U U}^{0}}{r_{U U}^{0}}
$$

$\mathrm{H}$ is then placed at various sites around the impurity, as shown in Figure 7a.

The energy of $\mathrm{H}$ absorption in a site adjacent to a chemical impurity was calculated for the impurities $\mathrm{C}, \mathrm{S}, \mathrm{Si}$ and $\mathrm{Ne}$. C, S, and $\mathrm{Si}$ were chosen to compare with available experimental observations of hydride formation in uranium metal where the concentration of these impurities was high. Ne was chosen as a control case to determine the extent to which direct impurity- $\mathrm{H}$ chemical interactions (i.e. bonding) may be partaking in the energy perturbation. For Ne these direct 
interactions are expected to be minimal. The deviation of the absorption energy for $\mathrm{H}$ from its pristine lattice value was decomposed into two major contributions: a strain component, which was calculated by freezing the atomic configurations as optimized for each particular case and then calculating the residual $\mathrm{H}$ absorption energy after the impurity atom is removed; and a binding component, which was calculated by subtracting the strain component from the total absorption energy. The strain component therefore reflects the energy gain due to $\mathrm{H}$ binding to the lattice in its now distorted configuration (Figure $7 \mathrm{~b}$ provides some idea of how the impurity atom distorts the local $\alpha$-U lattice), and the bonding component reflects how much the impurity atom modifies the nearby electronic structure of $U$ such that $\mathrm{H}$ bonding is enhanced.

We began our studies by studying $\mathrm{C}$ impurities and it was found that the sites shown in Figure $7 \mathrm{a}(\mathrm{i}, \mathrm{iii}, \mathrm{iv})$ were only slightly perturbed in energy (i.e. less than $0.05 \mathrm{eV}$ ), whereas the adjacent octahedral site (Figure 7a(ii)) was moresignificantly lowered in energy (by $0.15 \mathrm{eV}$ ). This effect makes the oct site now competitive towards hydrogen with the sqpy and tet sites. We note as an aside that this value is very close to the $0.14 \mathrm{eV}$ determined for $\mathrm{C}-\mathrm{H}$ interactions in $\mathrm{V}$ from experiments by Chang and Wert. ${ }^{37}$ Since the adjacent octahedral sites offer the maximal interaction between $\mathrm{H}$ and impurity atoms, our study of the impact of impurities on absorption will be focused on the Figure 7a(ii) configuration, in which the $\mathrm{H}$ and the impurity are thus located. 
The strain and bonding components for each of the impurity atoms have been tabulated in Table 2, and plotted graphically in Figure 8. Defects disturb the lattice in such a way that $\mathrm{H}$ absorption at the octahedral site becomes more favorable. In fact, the distortion can become so significant that the absorption of $\mathrm{H}$ into the $\alpha-\mathrm{U}$ lattice becomes exothermic (recall that binding at the pristine octahedral site is $0.53 \mathrm{eV}$ endothermic and note that the absorption energy is lowered by $>0.53 \mathrm{eV}$ when binding in the vicinity of $\mathrm{Si}$ ). The electronic and strain contributions are roughly equal in all cases. The fact that $\mathrm{Ne}$ correlates in the same fashion as $\mathrm{C}, \mathrm{S}$ and $\mathrm{Si}$ indicates that there is no special bonding occurring between the impurity atom and $\mathrm{H}$, but rather that the impurity atom distorts the geometric and electronic structure of $\mathrm{U}$ locally in such a way as to promote $\mathrm{H}$ binding. The correlation to atomic size is good, as indicated by the data in Table 2 . Lower binding energies mean that impurities can serve as trap sites for $\mathrm{H}$, and although we have only modeled single atom impurities, it can be envisaged that precipitates might act in the same way. Trap sites for $\mathrm{H}$ provide a means by which the local solubility of $\mathrm{H}$ can be enhanced over the few ppm allowed by the high chemical potential presented by the pristine lattice. It should be emphasized that strains calculated here to parameterize the impurities are local according to Eq. (1), and therefore not directly comparable with the strains applied globally in the previous section. It has been proposed previously that $\mathrm{H}$ and vacancies can mutually interact such that significant quantities of $\mathrm{H}$ can be absorbed into a lattice, while at the same time a 'superabundance' of vacancies can result - a likely precursor to void formation and subsequent mechanical failure. ${ }^{38} \mathrm{H}$ in the $\alpha-\mathrm{U}$ 
lattice possesses indicators of similar behavior, as determined by our calculations, with the energy of binding at a vacancy being exothermic relative to the $\mathrm{H}_{2}$ reference state. The chemical potential difference between the vacancy and pristine lattice amounts to $0.73 \mathrm{eV}$. The configuration of the vacancy site is shown in Figure 9. There is therefore a mutual stabilization of vacancies and lattice $H$ provided by this effect. It is not unlikely that hydride initiation may occur at such vacancy sites, where the lower heat of absorption and the creation of open surfaces within the material can ultimately favor the development of the higher-volume hydride phase. 


\section{Summary and Conclusions}

Using density functional theory, we have begun to identify critical steps and intermediates relevant to the hydriding of the orthorhombic phase of uranium metal $(\alpha-U)$. We focused on surface phenomena, absorption, transport in the bulk and trapping. It was found that the $\alpha-U(001)$ surface readily activates the $\mathrm{H}-\mathrm{H}$ bond leading to the adsorption of free hydrogen atoms. These adsorbed hydrogen atoms possess a negative charge of -0.5 e and contain the strongest bonds to Uranium as compared to sites in the bulk. Transport to bulk sites through a defect-free surface is endothermic, and contains an activation barrier that is higher than the experimentally observed Arrhenius activation energy. From this we conclude that hydrogen entry to the surface must occur at defect sites, such as grain boundaries, surface dislocations, precipitates, etc. The charge on $\mathrm{H}$ is $-0.5 \mathrm{e}$ in bulk sites as well as the surface. Our diffusion barrier for $\mathrm{H}$ in $\alpha-\mathrm{U}$ was consistent with experimental estimates for this value.

In addition to these points, we have shown that hydrogen-uranium bonding is strengthened at regions of (a) hydrostatic strain, (b) local strain introduced by impurity atoms, and (c) at vacancy sites. Thus each of these regions may act as trap sites, around which hydrogen atoms may gather and hydrides may nucleate. Further examination of these processes would require the development of an efficient atomistic potential treatment. In a subsequent paper we will present the results of $a b$ initio calculations 
of the U-H phase diagram, and pathways for the phase transformation of $\alpha-U$ saturated with hydrogen atoms to the $\mathrm{UH}_{3}$ phase. 


\section{References}

T. B. Flanagan, in Hydrides for Energy Storage (Pergamon Press, New York, 1977), p. 135.

M. Balooch and A. V. Hamza, J. Nuc. Mat. 230, 259 (1996).

E. Swissa, I. Jacob, U. Atzmony, N. Shamir, and M. H. Mintz, Surf. Sci. 223, 607 (1989).

M. W. Mallett and M. J. Trzeciak, Trans. ASM. 50, 981 (1958).

H. C. Mattraw, J. Phys. Chem. 59, 93 (1955).

C. Wolverton, V. Ozoliņš, and M. Asta, Phys. Rev. B 69, 144109 (2004).

D. E. Jiang and E. A. Carter, Phys. Rev. B 70, 64102 (2004).

C. Domain, R. Besson, and A. Legris, Acta Mat. 50, 3513 (2002).

Q. Xu and A. Van der Ven, Phys. Rev. B 76, 64207 (2007).

P. G. Sundell and G. Wahnström, Phys. Rev. B 70, 224301 (2004).

J. Greeley, W. P. Krekelberg, and M. Mavrikakis, Angew. Chem. Int. Ed. 43, 4296 (2004).

J. W. Turley and H. W. Rinn, Inorg. Chem. 8, 18 (1969).

A. C. Switendick, J. Less-Comm. Met. 88, 257 (1982).

K. Balasubramanian, W. J. Siekhaus, and W. Mc Lean II, J. Chem. Phys. 119, 5889 (2003).

J. L. Nie, H. Y. Xiao, X. T. Zu, and F. Gao, J. Phys.: Condens. Matter 20, 1 (2008).

G. Kresse and D. Joubert, Phys. Rev. B 59, 1758 (1999).

C. D. Taylor, Phys. Rev. B 77, 94119 (2008).

P. Söderlind, Phys. Rev. B 66, 85113 (2002).

G. Kresse and J. Furthmüller, Physical Review B 54, 11169 (1996).

J. P. Perdew, J. A. Chevary, S. H. Vosko, K. A. Jackson, M. R. Pederson, D. J. Singh, and C. Fiolhais, Physical Review B 46, 6671 (1992).

H. J. Monkhorst and J. D. Pack, Physical Review B 13, 5188 (1976).

M. Methfessel and A. T. Paxton, Physical Review B 40, 3616 (1989).

P. E. Blöchl, O. Jepsen, and O. K. Andersen, Physical Review B 49, 16223 (1994).

M. N. Huda and A. K. Ray, Phys. Rev. B 72, 85101 (2005).

I. N. Levine, Quantum Chemistry (Prentice HAll, Upper Saddle River, NY, 2000).

J. Bloch, J. Alloys. Comp. 361, 130 (2003).

P. Nordlander, S. Holloway, and J. K. Nørskov, Surf. Sci. 136, 59 (1984).

R. Bader, Atoms in Molecules: A Quantum Theory (Oxford University Press, New York, 1990).

G. Henkelman, A. Arnaldsson, and H. Jónsson, Comp. Mat. Sci. 36, 354 (2006).

L. E. Sutton ed., Tables of Interatomic Distances and Configurations in Molecules and Ions (Chem. Soc., London, 1958). 
31 J. C. Slater, J. Chem. Phys. 41, 3199 (1964).

32 A. Van der Ven and G. Ceder, Acta Mat. 52, 1223 (2002).

33 D. Crusset, F. Bernard, E. Sciora, and N. Gérard, J. Alloys. Comp. 204, 71 (1994).

34 M. E. Pronsato, C. Pistonesi, and A. Juan, J. Phys.: Condens. Matter 16, 6907 (2004).

35 G. Lu and E. Kaxiras, Phys. Rev. Lett. 94, 155501 (2005).

36 C. Zhang and A. Alavi, J. Am. Chem. Soc. 127, 9808 (2005).

37 H. Y. Chang and C. A. Wert, Acta. Met. 21, 1233 (1973).

38 Y. Fukai, J. Alloys. Comp. 356-357, 263 (2003). 


\section{Table Captions}

Table 1. Binding energies for $\mathrm{H}$ relative to the atomic reference state, $\mathrm{E}(\mathrm{H})$, or the molecular reference state, $\mathrm{E}\left(1 / 2 \mathrm{H}_{2}\right)$, at various surface, interstitial and trapping sites in $\alpha$-U lattice. Energies are given in $\mathrm{eV} / \mathrm{H}$ atom.

Table 2. Decomposition of binding energies, in $\mathrm{eV}$, into strain, $\mathrm{E}_{\mathrm{s}}$, and binding energies, $\mathrm{E}_{\mathrm{B}}$, for $\mathrm{H}$ absorbed adjacent to a given impurity in the lattice. The average bond-length distortion for uranium atoms around the impurity is given as a percentage, $\langle\varepsilon\rangle$. 


\section{Figure Captions}

Figure 1. A schematic cartoon showing $H$ interacting with the orthorhombic phase of $\alpha-U$ at surfaces, grain-boundaries, lattice-sites, vacancies, impurities and a hydride $\left(\beta-\mathrm{UH}_{3}\right)$ precipitate. In this paper we shall focus on a number of these interaction zones, and use strained lattices to simulate the binding of $\mathrm{H}$ at a grain boundary.

Figure 2. The $\alpha-\mathrm{U}$ crystal structure, provided as a reference for the discussion and results to follow.

Figure 3. Potential energy surface for $\mathrm{H}_{2}$ descending over the atop site with $\mathrm{H}$ atoms pointing towards three fold sites.

Figure 4. High symmetry adsorption sites on the $\alpha-\mathrm{U}(001)$ surface (a) atop, (b) bridge-1, (c) bridge-2, (d) e-hcp, and (e) e-fcc. Large gray atoms are U (some sub-surface atoms are shown), and small white atoms are $\mathrm{H}$ with bonds indicating shortest $\mathrm{U}-\mathrm{H}$ contacts.

Figure 5. Interstitial sites for $\mathrm{H}$ in $\alpha-\mathrm{U}$ the lattice: (a) octahedral site, (b) square pyramidal site, (c) tetrahedral site, (d) diffusion barrier site bridging sqp and tetrahedral sites.

Figure 6. Energy of $\mathrm{H}$ absorption as a function of hydrostatic strain.

Figure 7. (a) Adjacent sites used to model the effect of interstitial impurities on the absorption of nearby $\mathrm{H}$ atoms, and (b) Local distortion of the lattice in the vicinity of the Ne impurity atom sitting in the octahedral site - note how the structural distortion of the defect is almost completely screened by the

first shell of $\mathrm{U}$ atoms. 
Figure $8 . \mathrm{H}$ binding energies in the vicinity of impurity sites decomposed into accommodation (strain reduction) and electronic terms (binding enhancement), plotted against the mean percent strain induced in the first coordination shell of the impurity atom.

Figure 9. The geometric environment of $\mathrm{H}$ adsorbed in a vacancy site in $\alpha-\mathrm{U}$. 


\section{Tables}

Table 1. Binding energies for $\mathrm{H}$ relative to the atomic reference state, $\mathrm{E}(\mathrm{H})$, or the molecular reference state, $E\left(1 / 2 \mathrm{H}_{2}\right)$, at various surface, interstitial and trapping sites in $\alpha-U$ lattice. Energies are given in $\mathrm{eV} / \mathrm{H}$ atom.

\begin{tabular}{lll|lll}
\hline Site & $\mathrm{E}(\mathrm{H})$ & $\mathrm{E}\left(1 / 2 \mathrm{H}_{2}\right)$ & Site & $\mathrm{E}(\mathrm{H})$ & $\mathrm{E}\left(1 / 2 \mathrm{H}_{2}\right)$ \\
\hline Atop & -1.86 & 0.44 & Tetrahedral & -1.89 & 0.41 \\
Bridge-1 & -2.63 & -0.33 & Octahedral & -1.72 & 0.58 \\
Bridge-2 & -2.59 & -0.29 & Square Pyramid & -1.91 & 0.39 \\
e-hcp & -2.78 & -0.48 & Planar (diff. TS) & -1.29 & 1.01 \\
e-fcc & -2.83 & -0.53 & Vacancy & -2.64 & -0.34 \\
Entry-d & -2.26 & 0.04 & S (oct-oct) & -1.97 & 0.33 \\
Entry-e & -2.08 & 0.22 & C (oct-oct) & -1.88 & 0.42 \\
H & & -0.11 & Ne (oct-oct) & -2.09 & 0.21 \\
& & & Si (oct-oct) & -2.34 & -0.04 \\
\hline
\end{tabular}


Table 2. Decomposition of binding energies, in $\mathrm{eV}$, into strain, $\mathrm{E}_{\mathrm{s}}$, and binding energies, $\mathrm{E}_{\mathrm{B}}$, for $\mathrm{H}$ absorbed adjacent to a given impurity in the lattice. The average bond-length distortion for uranium atoms around the impurity is given as a percentage, $\langle\varepsilon\rangle$.

\begin{tabular}{llllll}
\hline Impurity & $\mathrm{R}_{\text {cov }}, \mathrm{pm}$ & $\langle\varepsilon, \%$ & $\mathrm{E}_{\mathrm{s}}$ reduction & $\mathrm{E}_{\mathrm{B}}$ gain & Net E gain \\
\hline $\mathrm{C}$ & 77 & 11 & 0.10 & 0.05 & 0.16 \\
$\mathrm{~S}$ & 110 & 14 & 0.12 & 0.13 & 0.25 \\
$\mathrm{Ne}$ & 125 & 17 & 0.17 & 0.20 & 0.37 \\
$\mathrm{Si}$ & 127 & 21 & 0.29 & 0.33 & 0.62 \\
\hline
\end{tabular}


Figures

Figure 1. A schematic cartoon showing $H$ interacting with the orthorhombic phase of $\alpha-U$ at surfaces, grain-boundaries, lattice-sites, vacancies, impurities and a hydride $\left(\beta-\mathrm{UH}_{3}\right)$ precipitate. In this paper we shall focus on a number of these interaction zones, and use strained lattices to simulate the binding of $\mathrm{H}$ at a grain boundary.

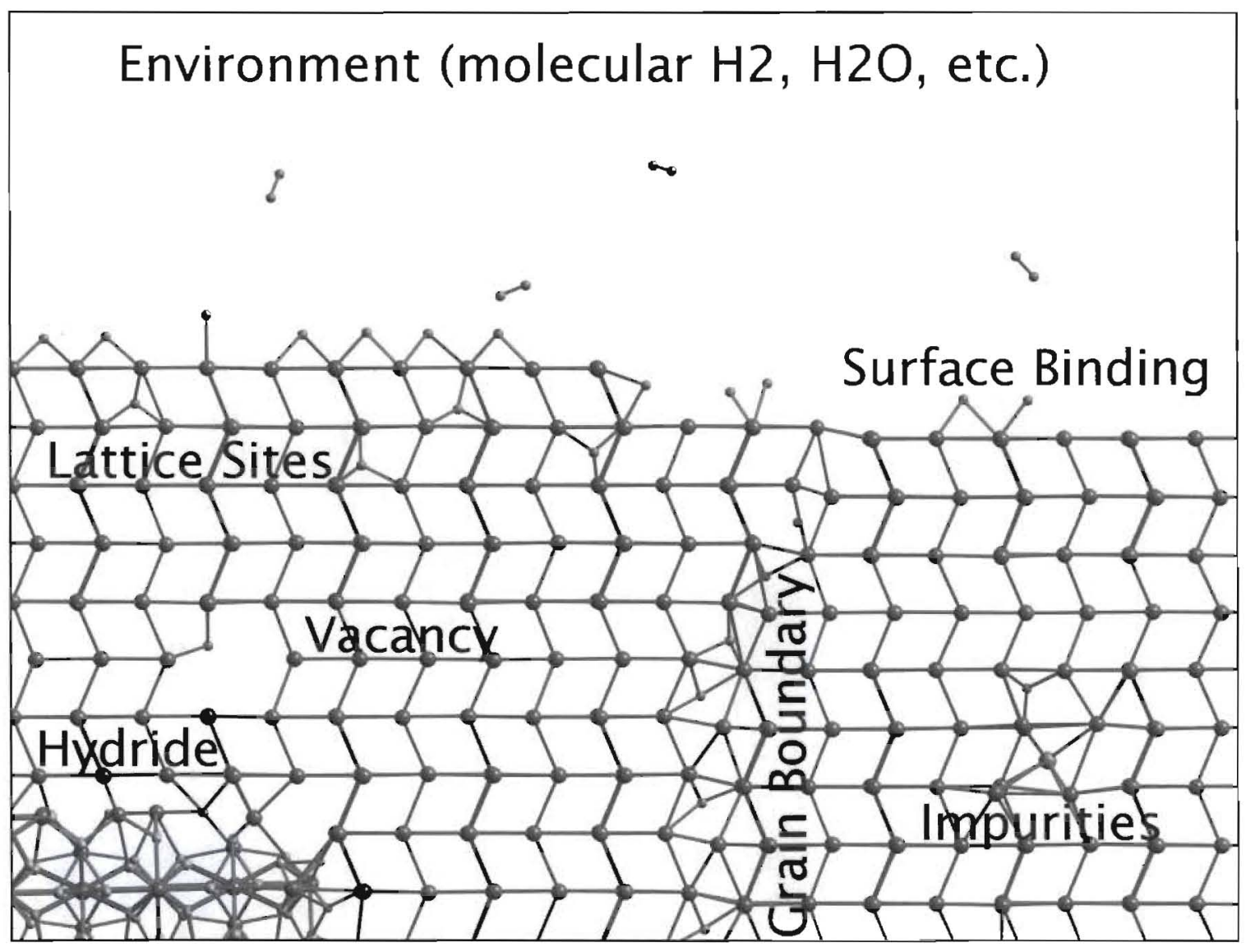


Figure 2. The $\alpha-U$ crystal structure, provided as a reference for the discussion and results to follow.

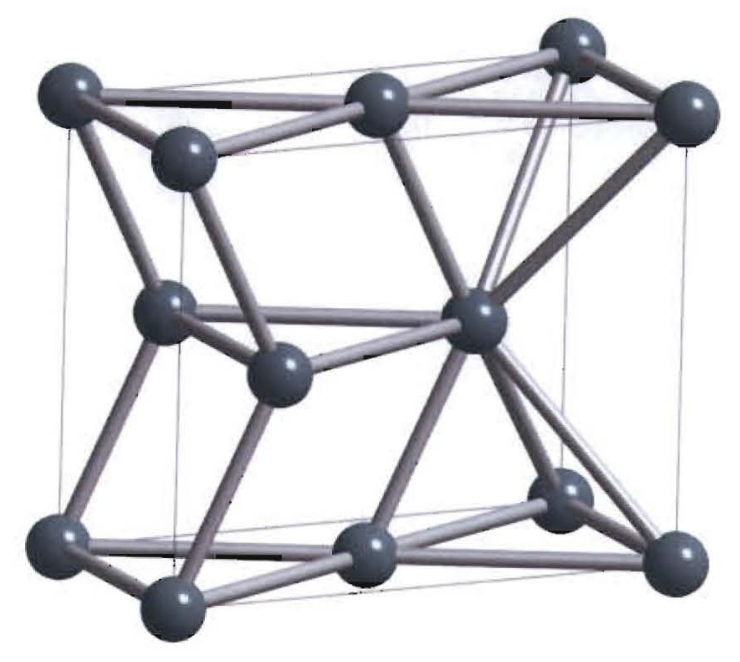


Figure 3. Potential energy surface for $\mathrm{H}_{2}$ descending over the atop site with $\mathrm{H}$ atoms pointing towards three fold sites.

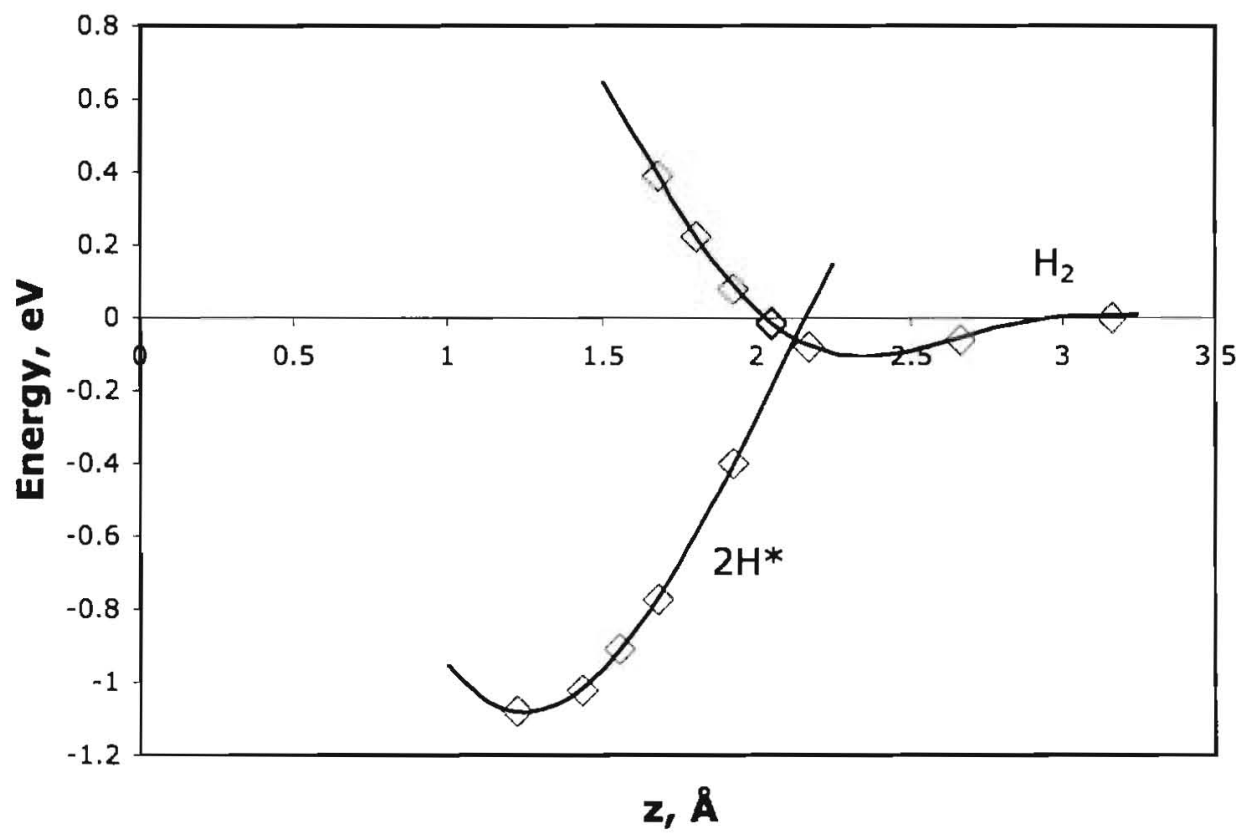


Figure 4. High symmetry adsorption sites on the $\alpha-U$ (001) surface (a) atop, (b) bridge-1, (c) bridge-2, (d) e-hcp, and (e) e-fcc. Large gray atoms are U (some sub-surface atoms are shown), and small white atoms are $\mathrm{H}$ with bonds indicating shortest $\mathrm{U}-\mathrm{H}$ contacts.

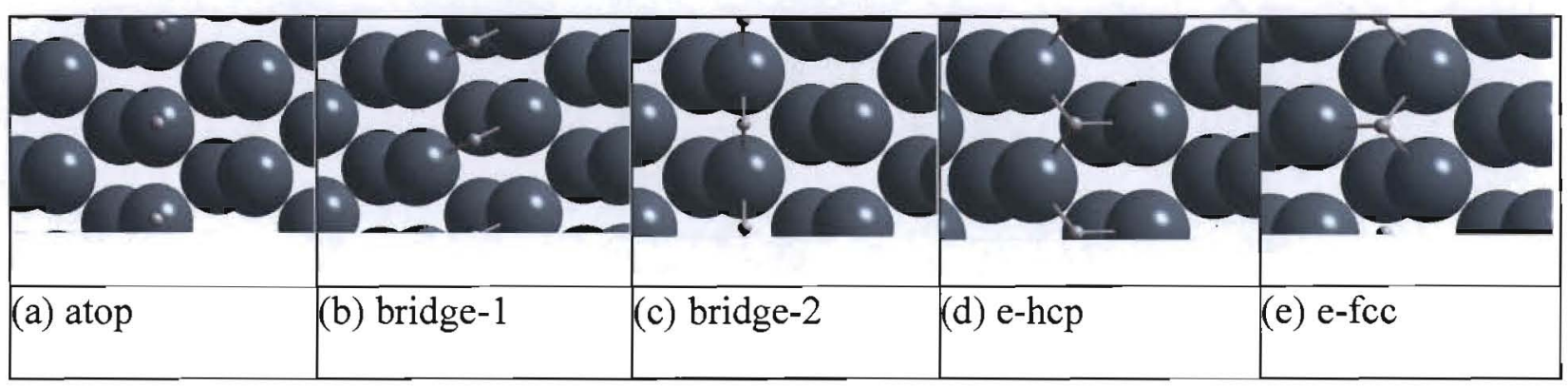


Figure 5. Interstitial sites for $H$ in $\alpha-U$ the lattice: (a) octahedral site, (b) square pyramidal site, (c) tetrahedral site, (d) diffusion barrier site bridging sqp and tetrahedral sites.

(a) (c) (d)


Figure 6. Energy of $\mathrm{H}$ absorption as a function of hydrostatic strain.

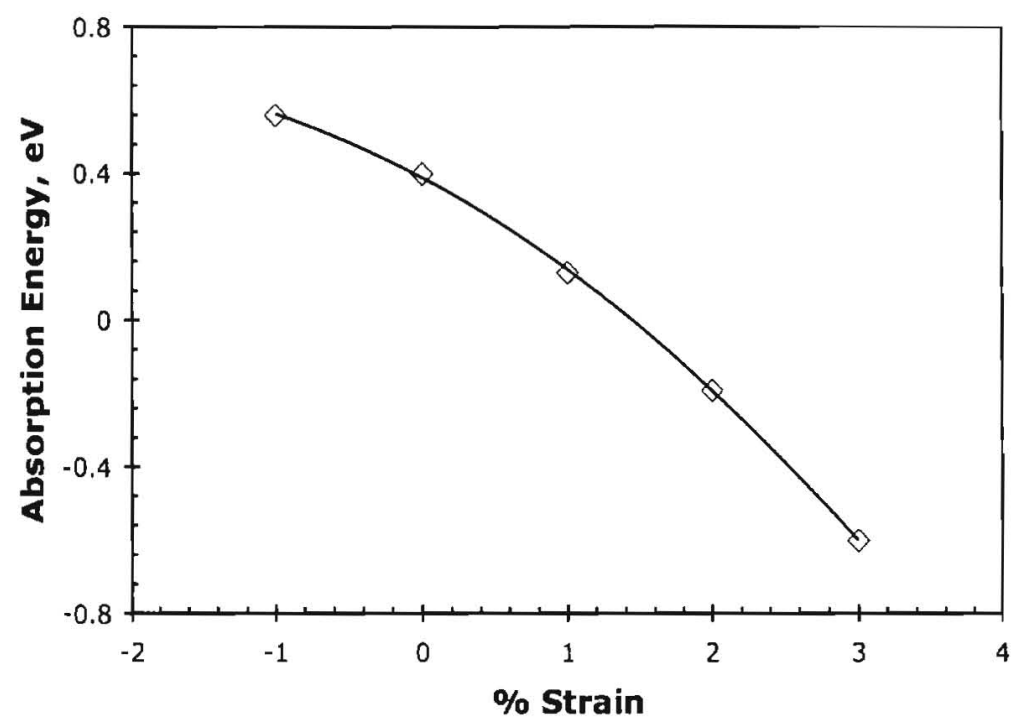


Figure 7. (a) Adjacent sites used to model the effect of interstitial impurities on the absorption of nearby $\mathrm{H}$ atoms, and (b) Local distortion of the lattice in the vicinity of the Ne impurity atom sitting in the octahedral site - note how the structural distortion of the defect is almost completely screened by the first shell of $\mathrm{U}$ atoms.

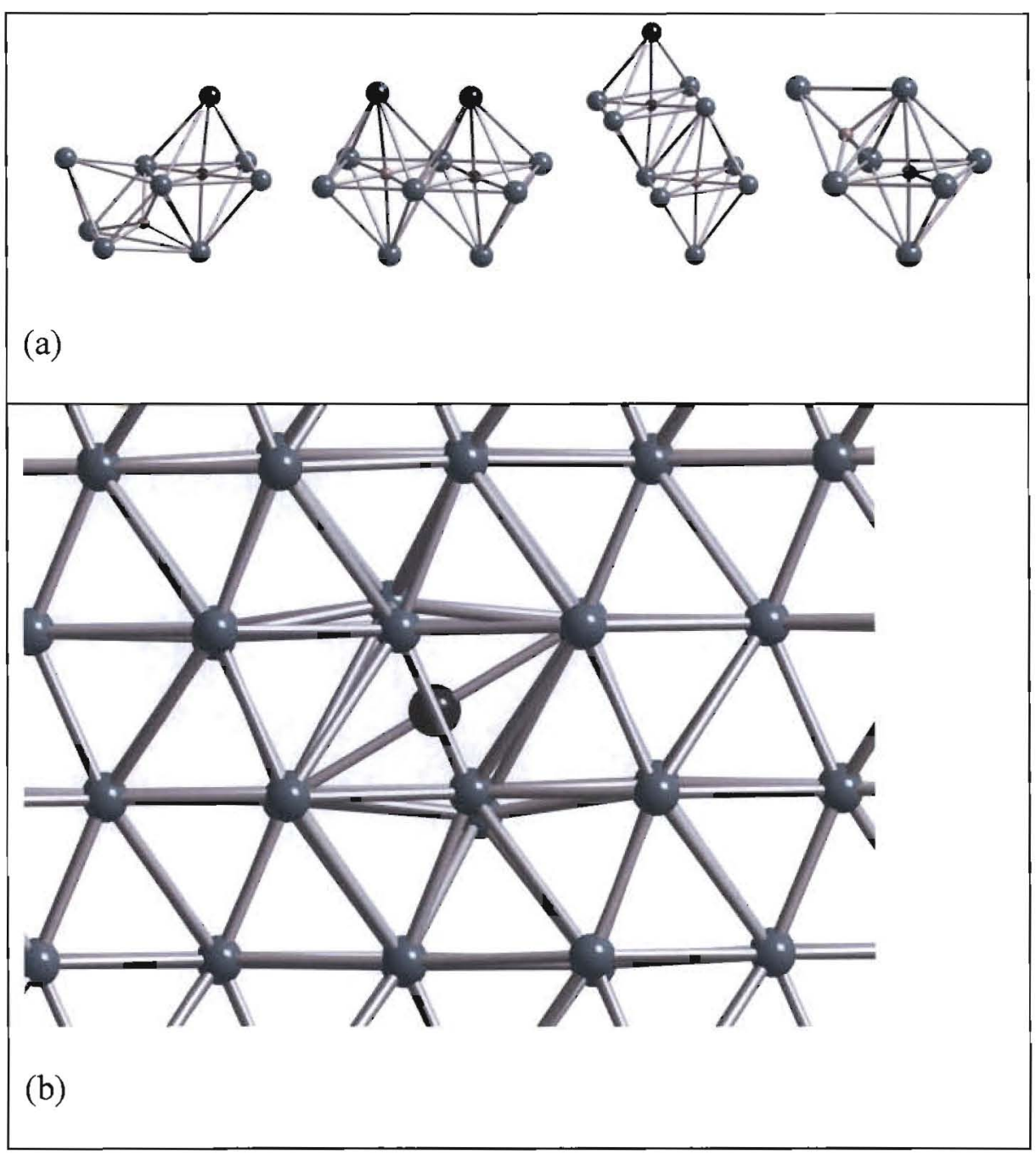


Figure 8 . H binding energies in the vicinity of impurity sites decomposed into accommodation (strain reduction) and electronic terms (binding enhancement), plotted against the mean percent strain induced in the first coordination shell of the impurity atom.

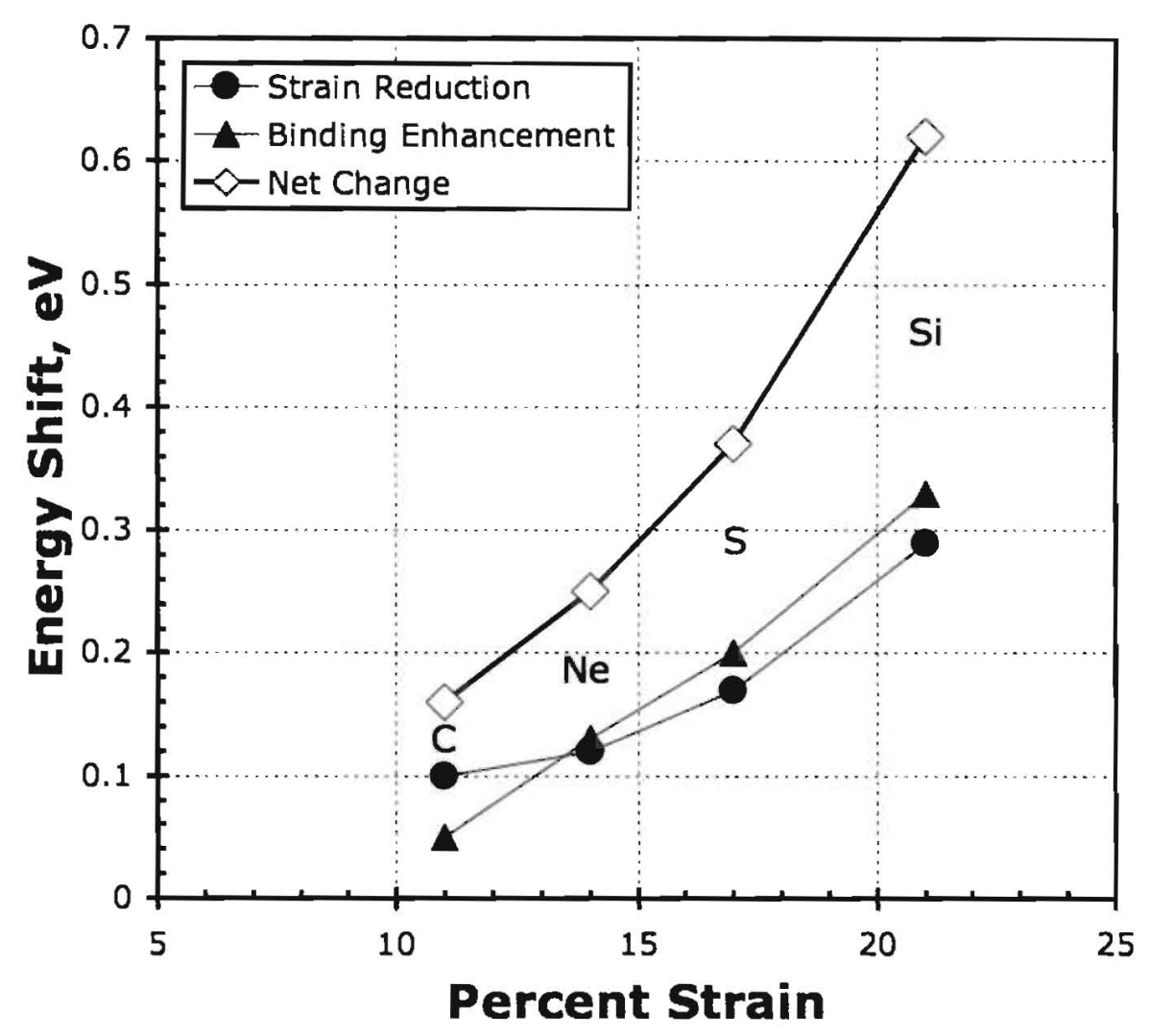


Figure 9. The geometric environment of $\mathrm{H}$ adsorbed in a vacancy site in $\alpha-\mathrm{U}$.

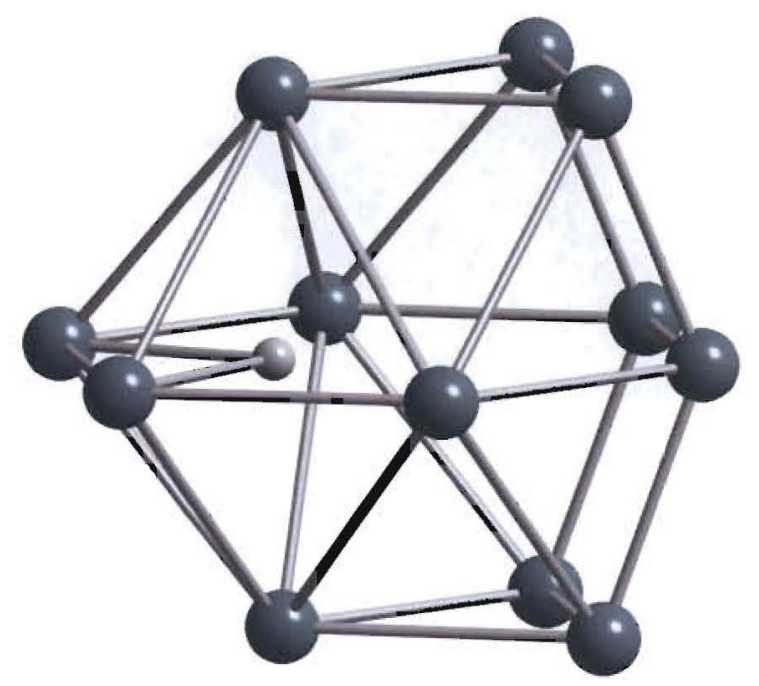

